\title{
SUPERSTABILITY OF ADJOINTABLE MAPPINGS ON HILBERT $C^{*}$-MODULES
}

\author{
Michael Frank, Pasc Găvruţa and Mohammad Sal Moslehian
}

Dedicated to the Memory of Professor D. S. Mitrinović

\begin{abstract}
We define the notion of $\varphi$-perturbation of a densely defined adjointable mapping and prove that any such mapping $f$ between Hilbert $\mathcal{A}$-modules over a fixed $C^{*}$-algebra $\mathcal{A}$ with densely defined corresponding mapping $g$ is $\mathcal{A}$-linear and adjointable in the classical sense with adjoint $g$. If both $f$ and $g$ are everywhere defined then they are bounded. Our work concerns with the concept of Hyers-Ulam-Rassias stability originated from the Th.M. Rassias' stability theorem that appeared in his paper [On the stability of the linear mapping in Banach spaces, Proc. Amer. Math. Soc. 72 (1978), 297-300]. We also indicate interesting complementary results in the case where the Hilbert $C^{*}$-modules admit non-adjointable $C^{*}$-linear mappings.
\end{abstract}

\section{INTRODUCTION}

We say a functional equation $(\mathcal{E})$ is stable if any function $g$ approximately satisfying the equation $(\mathcal{E})$ is near to an exact solution of $(\mathcal{E})$. The equation $(\mathcal{E})$ is called superstable if every approximate solution of $(\mathcal{E})$ is indeed a solution (see [5] for another notion of superstability namely superstability modulo the bounded functions). More than a half century ago, S.M. Ulam [23] proposed the first stability problem which was partially solved by D.H. Hyers [10] in the framework of Banach spaces. Later, T. Aoki 3 proved the stability of the additive mapping and Th.M. Rassias [20] proved the stability of the linear mapping for mappings $f$ from a normed space into a Banach space such that the norm of the Cauchy difference $f(x+y)-f(x)-f(y)$ is bounded by the expression $\varepsilon\left(\|x\|^{p}+\|y\|^{p}\right)$ for some $\varepsilon \geq 0$, for some $0 \leq p<1$ and for all $x, y$. The terminology "HyersUlam-Rassias stability" was indeed originated from Th.M. Rassias's paper [20]. In 1994, a further generalization was obtained by P. Găvruţa [9], in which he replaced the bound $\varepsilon\left(\|x\|^{p}+\|y\|^{p}\right)$ by a general control function $\varphi(x, y)$. This terminology can be applied to functional equations and mappings on various generalized notions of Hilbert spaces; see 1, 2, 4. We refer the interested reader to monographs [6, 17, 11, 13, 19, 22, and references therein for more information.

The notion of Hilbert $C^{*}$-module is a generalization of the notion of Hilbert space. This object was first used by I. Kaplansky [14. Interacting with the theory of

2000 Mathematics Subject Classification: Primary 46L08; Secondary 47B48, 39B52, 46L05 Keywords and Prases: Hyers-Ulam-Rassias stability, superstability, Hilbert $C^{*}$-module, $C^{*}$-algebra, $\varphi$-perturbation of an adjointable mapping. 
operator algebras and including ideas from non-commutative geometry it progresses and produces results and new problems attracting attention, see [8, 15, 18.

Let $\mathcal{A}$ be a $C^{*}$-algebra and $\mathcal{X}$ be a complex linear space, which is a right $\mathcal{A}$-module with a scalar multiplication satisfying $\lambda(x a)=x(\lambda a)=(\lambda x) a$ for $x \in \mathcal{X}, a \in \mathcal{A}, \lambda \in$ $\mathbb{C}$. The space $\mathcal{X}$ is called a (right) pre-Hilbert $\mathcal{A}$-module if there exists an $\mathcal{A}$-inner product $\langle.,\rangle:. \mathcal{X} \times \mathcal{X} \rightarrow \mathcal{A}$ satisfying

(i) $\langle x, x\rangle \geq 0$ and $\langle x, x\rangle=0$ if and only if $x=0$;

(ii) $\langle x, y+\lambda z\rangle=\langle x, y\rangle+\lambda\langle x, z\rangle$;

(iii) $\langle x, y a\rangle=\langle x, y\rangle a$;

(iv) $\langle x, y\rangle^{*}=\langle y, x\rangle$;

for all $x, y, z \in \mathcal{X}, \lambda \in \mathbb{C}, a \in \mathcal{A}$. The pre-Hilbert module $\mathcal{X}$ is called a (right) Hilbert $\mathcal{A}$-module if it is complete with respect to the norm $\|x\|=\|\langle x, x\rangle\|^{1 / 2}$. Left Hilbert $\mathcal{A}$-modules can be defined in a similar way. Two typical examples are

(I) Every inner product space is a left pre-Hilbert $\mathbb{C}$-module.

(II) Let $\mathcal{A}$ be a $C^{*}$-algebra. Then every norm-closed right ideal $I$ of $\mathcal{A}$ is a Hilbert $\mathcal{A}$-module if one defines $\langle a, b\rangle=a^{*} b \quad(a, b \in I)$.

A mapping $f: \mathcal{X} \rightarrow \mathcal{Y}$ between Hilbert $\mathcal{A}$-modules is called adjointable if there exists a mapping $g: \mathcal{Y} \rightarrow \mathcal{X}$ such that $\langle f(x), y\rangle=\langle x, g(y)\rangle$ for all $x \in \mathcal{D}(f) \subseteq$ $\mathcal{X}, y \in \mathcal{D} \subseteq \mathcal{Y}$. Throughout the paper, we assume that $f$ and $g$ are both everywhere defined or both densely defined. The unique mapping $g$ is denoted by $f^{*}$ and is called the adjoint of $f$.

An $\mathcal{A}$-linear bounded operator $K$ on a Hilbert $\mathcal{A}$-module $\mathcal{X}$ is called "compact" if it belongs to the norm-closed linear span of the set of all elementary operators $\theta_{x, y}(x, y \in \mathcal{X})$ defined by $\theta_{x, y}(z)=x\langle y, z\rangle(z \in \mathcal{X})$.

In this paper, we prove the superstability of adjointable mappings on Hilbert $C^{*}$-modules in the spirit of Hyers-Ulam-Rassias and indicate interesting complementary results in the case where the Hilbert $C^{*}$-modules admit non-adjointable $C^{*}$-linear mappings.

\section{MAIN RESULTS}

Throughout this section, $\mathcal{A}$ denotes a $C^{*}$-algebra, $\mathcal{X}$ and $\mathcal{Y}$ denote Hilbert $\mathcal{A}$-modules, and $\varphi: \mathcal{X} \times \mathcal{Y} \rightarrow[0, \infty)$ is a function. We start our work with the following definition.

Definition 2.1. A (not necessarily linear) mapping $f: \mathcal{X} \rightarrow \mathcal{Y}$ is called a $\varphi$ perturbation of an adjointable mapping if there exists a (not necessarily linear) corresponding mapping $g: \mathcal{Y} \rightarrow \mathcal{X}$ such that

$$
\|\langle f(x), y\rangle-\langle x, g(y)\rangle\| \leq \varphi(x, y) \quad(x \in \mathcal{D}(f) \subseteq \mathcal{X}, y \in \mathcal{D}(g) \subseteq \mathcal{Y}) .
$$

To prove our main result, we need the following known lemma (cf. [15, p. 8]) that we prove it for the sake of completeness. 
Lemma 2.2. Every densely defined adjointable mapping between Hilbert $C^{*}{ }^{-}$modules over a fixed $C^{*}$-algebra $\mathcal{A}$ is $\mathcal{A}$-linear. If the adjointable mapping is everywhere defined then it is bounded.

Proof. Let $f: \mathcal{X} \rightarrow \mathcal{Y}$ and $g: \mathcal{Y} \rightarrow \mathcal{X}$ be a pair of densely defined adjointable mappings between two Hilbert $C^{*}$-modules $\mathcal{X}$ and $\mathcal{Y}$. For every $x_{1}, x_{2}, x_{3} \in \mathcal{D}(f) \subseteq$ $\mathcal{X}$, every $y \in \mathcal{D}(g) \subseteq \mathcal{Y}$, every $\lambda \in \mathbb{C}$, every $a \in \mathcal{A}$ the following equality holds:

$$
\begin{aligned}
\left\langle f\left(\lambda x_{1}+x_{2}+x_{3} a\right), y\right\rangle & =\left\langle\lambda x_{1}+x_{2}+x_{3} a, g(y)\right\rangle \\
& =\lambda\left\langle x_{1}, g(y)\right\rangle+\left\langle x_{2}, g(y)\right\rangle+a^{*}\left\langle x_{3}, g(y)\right\rangle \\
& =\lambda\left\langle f\left(x_{1}\right), y\right\rangle+\left\langle f\left(x_{2}\right), y\right\rangle+a^{*}\left\langle f\left(x_{3}\right), y\right\rangle \\
& =\left\langle\lambda f\left(x_{1}\right)+f\left(x_{2}\right)+f\left(x_{3}\right) a, y\right\rangle .
\end{aligned}
$$

By the density of the domain of $g$ in $\mathcal{Y}$ the equality yields the $\mathcal{A}$-linearity of $f$.

Now, suppose $f$ and $g$ to be everywhere defined on $\mathcal{X}$ and $\mathcal{Y}$, respectively. For each $x$ in the unit sphere of $\mathcal{X}$, define $\tau_{x}: \mathcal{Y} \rightarrow \mathcal{A}$ by $\tau_{x}(y)=\langle f(x), y\rangle=\langle x, g(y)\rangle$. Then $\left\|\tau_{x}(y)\right\| \leq\|x\|\|g(y)\| \leq\|g(y)\|$ for any $x$ from the unit ball. By the BanachSteinhaus theorem we conclude that the set $\left\{\left\|\tau_{x}\right\|: x \in \mathcal{X},\|x\| \leq 1\right\}$ is bounded. Due to the equality $\|f(x)\|=\sup _{\|y\| \leq 1}\|\langle f(x), y\rangle\|=\sup _{\|y\|=1}\left\|\tau_{x}(y)\right\|=\left\|\tau_{x}\right\|$ the mapping $f$ has to be bounded.

Theorem 2.3. Let $f: \mathcal{X} \rightarrow \mathcal{Y}$ be a $\varphi$-perturbation of an adjointable mapping with corresponding mapping $g: \mathcal{Y} \rightarrow \mathcal{X}$. Suppose that the mappings $f$ and $g$ are everywhere defined on the respective Hilbert $C^{*}$-modules. Furthermore, suppose that for some sequence $\left\{c_{n}\right\}$ of non-zero complex numbers either both the conditions (2.2) and (2.3) or both the conditions (2.4) and (2.5) below hold for the perturbation bound mapping $\varphi(x, y)$ :

$$
\begin{array}{ll}
\lim _{n \rightarrow \infty}\left|c_{n}\right|^{-1} \varphi\left(c_{n} x, y\right)=0 & (x \in \mathcal{X}, y \in \mathcal{Y}) \\
\lim _{n \rightarrow \infty}\left|c_{n}\right|^{-1} \varphi\left(x, c_{n} y\right)=0 & (x \in \mathcal{X}, y \in \mathcal{Y}), \\
\lim _{n \rightarrow \infty}\left|c_{n}\right| \varphi\left(c_{n}^{-1} x, y\right)=0 & (x \in \mathcal{X}, y \in \mathcal{Y}) \\
\lim _{n \rightarrow \infty}\left|c_{n}\right| \varphi\left(x, c_{n}^{-1} y\right)=0 & (x \in \mathcal{X}, y \in \mathcal{Y}) .
\end{array}
$$

Then $f$ is adjointable. In particular, $f$ is bounded, continuous and $\mathcal{A}$-linear, as well as its adjoint is $g$.

Proof. Let $\lambda \in \mathbb{C}$ be an arbitrary number. Replacing $x$ by $\lambda x$ in (2.1), we get

$$
\|\langle f(\lambda x), y\rangle-\langle\lambda x, g(y)\rangle\| \leq \varphi(\lambda x, y),
$$

and since a multiplication of (2.1) by $|\lambda|$ yields

$$
\|\langle\lambda f(x), y\rangle-\langle\lambda x, g(y)\rangle\| \leq|\lambda| \varphi(x, y)
$$


we obtain

$$
\|\langle f(\lambda x), y\rangle-\langle\lambda f(x), y\rangle\| \leq \varphi(\lambda x, y)+|\lambda| \varphi(x, y)
$$

If (2.3) holds, we take $c_{n} y$ instead $y$ in (2.6) to get

$$
\|\langle f(\lambda x), y\rangle-\langle\lambda f(x), y\rangle\| \leq\left|c_{n}\right|^{-1} \varphi\left(\lambda x, c_{n} y\right)+|\lambda|\left|c_{n}\right|^{-1} \varphi\left(x, c_{n} y\right)
$$

and, as $n \rightarrow \infty$, we obtain

$$
\langle f(\lambda x), y\rangle=\langle\lambda f(x), y\rangle \quad(x \in \mathcal{X}, y \in \mathcal{Y}) .
$$

If (2.5) holds, we take $c_{n}^{-1} y$ instead $y$ in (2.6) and we arrive also at (2.7). Therefore,

$$
f(\lambda x)=\lambda f(x) \quad(x \in \mathcal{X}, \lambda \in \mathbb{C}) .
$$

If (2.2) holds, we take $c_{n} x$ instead $x$ in (2.1) to get

$$
\left\|\left\langle f\left(c_{n} x\right), y\right\rangle-\left\langle c_{n} x, g(y)\right\rangle\right\| \leq \varphi\left(c_{n} x, y\right)
$$

and, by (2.7), we obtain

$$
\|\langle f(x), y\rangle-\langle x, g(y)\rangle\| \leq\left|c_{n}\right|^{-1} \varphi\left(c_{n} x, y\right)
$$

Taking the limit as $n \rightarrow \infty$ we conclude that

$$
\langle f(x), y\rangle=\langle x, g(y)\rangle \quad(x \in \mathcal{X}, y \in \mathcal{Y}) .
$$

Hence $f$ is adjointable and admits the mapping $g$ as its adjoint.

Alternatively, if (2.4) holds, we take $c_{n}^{-1} x$ instead $x$ in (2.6) and arrive at the same conclusion (2.9). By Lemma 2.2 the mapping $f$ is $\mathcal{A}$-linear and bounded with the adjoint $g$.

Using the sequence $c_{n}=2^{n}$ we get the following results.

Corollary 2.4. If $f: \mathcal{X} \rightarrow \mathcal{Y}$ is an everywhere defined $\varphi$-perturbation of an adjointable mapping, where $\varphi(x, y)=\varepsilon\|x\|^{p}\|y\|^{q}(\alpha>0, p \neq 1, q \neq 1)$, then $f$ is adjointable and hence a bounded $C^{*}$-linear mapping.

Corollary 2.5. If $f: \mathcal{X} \rightarrow \mathcal{Y}$ is an everywhere defined $\varphi$-perturbation of an adjointable mapping, where $\varphi(x, y)=\varepsilon_{1}\|x\|^{p}+\varepsilon_{2}\|y\|^{q}\left(\varepsilon_{1} \geq 0, \varepsilon_{2} \geq 0, p \neq 1, q \neq 1\right)$, then $f$ is adjointable and hence a bounded $C^{*}$-linear mapping.

We would like to point out that the proof of Theorem 2.3 works equally well in the case that the functions $f$ and $g$ are well-defined merely on norm-dense subsets of $\mathcal{X}$ and $\mathcal{Y}$, respectively. This case covers the situation of pairs of adjoint to each other, densely defined $\mathcal{A}$-linear operators between pairs of Hilbert $\mathcal{A}$-modules. However, since boundedness cannot be demonstrated, in general, in that case we arrive at the following statement:

Theorem 2.6. Let $f: \mathcal{X} \rightarrow \mathcal{Y}$ be a $\varphi$-perturbation of an adjointable mapping with corresponding mapping $g: \mathcal{Y} \rightarrow \mathcal{X}$. Suppose, that the mappings $f$ and $g$ are densely defined on the respective Hilbert $C^{*}$-modules. Furthermore, suppose that for the perturbation bound mapping $\varphi(x, y)$ either both the conditions (2.2) and (2.3), or both the conditions (2.4) and (2.5) hold. Then $f$ is adjointable. In particular, $f$ is $\mathcal{A}$-linear, as well as its adjoint is $g$. 
Corollary 2.7. The equation $f(x)^{*} y=x g(y)^{*} \quad(x \in \mathcal{I}, y \in \mathcal{J})$ is superstable, where

$f: \mathcal{I} \rightarrow \mathcal{J}$ and $g: \mathcal{J} \rightarrow \mathcal{I}$ are adjoint to each other, densely defined $\mathcal{A}$-linear mappings between right ideals $\mathcal{I}, \mathcal{J}$ of $\mathcal{A}$.

The critical case of $\varphi$-perturbations is that one where the function $\varphi$ satisfies neither the pair of conditions (i) and (ii), nor the pair of conditions (i') and (ii'). We demonstrate that there may exist $\varphi$-perturbed bounded $C^{*}$-linear mappings $f$ on certain types of Hilbert $C^{*}$-modules $\mathcal{X}$ over certain $C^{*}$-algebras $\mathcal{A}$ which are not adjointable. Moreover, any non-adjointable bounded $C^{*}$-linear mapping $f$ on suitably selected Hilbert $C^{*}$-modules $\mathcal{X}$ can be $\varphi$-perturbed by "compact" operators on $\mathcal{X}$ using this type of perturbation functions.

Proposition 2.8. Let $\mathcal{X}$ be a Hilbert $\mathcal{A}$-module over a given $C^{*}$-algebra $\mathcal{A}$. Suppose there exists a non-adjointable bounded $\mathcal{A}$-linear mapping $f: \mathcal{X} \rightarrow \mathcal{X}$, (so $\mathcal{X}$ cannot be self-dual by [15, 8]). Then there exist (at least countably many) positive constants $c_{\alpha}$ and respective "compact" $\mathcal{A}$-linear operators $K_{\alpha}: \mathcal{X} \rightarrow \mathcal{X}(\alpha \in I)$ such that $f$ is $\phi$-perturbed for a function $\phi(x, y)=c_{\alpha} \cdot\|x\| \cdot\|y\|$ and for $g=K_{\alpha}^{*}$.

Proof. By results of Huaxin Lin [16, [17, Thm. 1.5], the Banach algebra $\operatorname{End}_{\mathcal{A}}(\mathcal{X})$ of all bounded $\mathcal{A}$-linear mappings on $\mathcal{X}$ is the left multiplier algebra of the $\mathrm{C}^{*}$ algebra $K_{\mathcal{A}}(\mathcal{X})$ of all "compact" $\mathcal{A}$-linear operators on $\mathcal{X}$. Since $E n d_{\mathcal{A}}(\mathcal{X})$ is the completion of $K_{\mathcal{A}}(\mathcal{X})$ with respect to the left strict topology defined by the set of semi-norms $\left\{\|\cdot K\|: K \in K_{\mathcal{A}}(\mathcal{X})\right\}$, there exists a bounded net $\left\{K_{\alpha}: \alpha \in I\right\}$ of "compact" operators such that the set $\left\{K_{\alpha} K: \alpha \in I\right\}$ converges with respect to the operator norm to $f K$ for any single "compact" operator $K$. Therefore,

$$
0=\lim _{\alpha \in I}\left\|\left\langle\left(f K-K_{\alpha} K\right)(x), y\right\rangle\right\|=\lim _{\alpha \in I}\left\|\left\langle\left(f-K_{\alpha}\right) K(x), y\right\rangle\right\|
$$

for any "compact" operator $K$. However, the set $\left\{K(x): K \in K_{\mathcal{A}}(\mathcal{X}), x \in \mathcal{X}\right\}$ is norm-dense in $\mathcal{X}$, hence

$$
\left\|\langle f(x), y\rangle-\left\langle K_{\alpha}(x), y\right\rangle\right\| \leq\left\|f-K_{\alpha}\right\| \cdot\|x\| \cdot\|y\|
$$

for any $x, y \in \mathcal{X}$ and any $\alpha \in I$. Setting $c_{\alpha}=\left\|f-K_{\alpha}\right\|$ for any fixed index $\alpha$ and taking into account the adjointability of the operators $\left\{K_{\alpha}\right\}$ we arrive at the desired result.

Corollary 2.9. Let $\mathcal{X}$ be a Hilbert $\mathcal{A}$-module over a given $C^{*}$-algebra $\mathcal{A}$. Suppose there exists a non-adjointable bounded $\mathcal{A}$-linear mapping $f: \mathcal{X} \rightarrow \mathcal{X}$. Then there does not exist any $\varphi$-perturbation of $f$ such that $\varphi(x, y)$ satisfies either both the conditions (2.2) and (2.3) or both the conditions (2.4) and (2.5).

\section{REFERENCES}

[1] M. Amyari: Stability of $C^{*}$-inner products, J. Math. Anal. Appl. 322 (2006), 214-218. 
[2] M. Amyari, M. S. Moslehian: Stability of derivations on Hilbert $C^{*}$-modules, Topological Algebras and Applications, 31-39, Contemp. Math. 724, 2007.

[3] T. AOKI: On the stability of the linear transformationin Banach spaces, J. Math. Soc. Japan 2 (1950), 64-66.

[4] C. BAAK, H. Y. Chu, M. S. Moslehian: On linear $n$-inner product preserving mappings, Math. Inequal. Appl., 9 (2006), no. 3, 453-464.

[5] J. BAKeR: The stability of the cosine equation, Proc. Amer. Math. Soc. 74 (1979), 242-246.

[6] S. CzerwiK (ED.): Stability of Functional Equations of Ulam-Hyers-Rassias Type, Hadronic Press Inc., Palm Harbor, Florida, 2003.

[7] S. Czerwik: Functional equations and inequalities in several variables, World Scientific Publishing Co., Inc., River Edge, NJ, 2002.

[8] M. Frank: Geometrical aspects of Hilbert $C^{*}$-modules, Positivity 3 (1999), 215-243.

[9] P. Găvruţa: A generalization of the Hyers-Ulam-Rassias stability of approximately additive mappings, J. Math. Anal. Appl. 184 (1994), 431-436.

[10] D. H. Hyers: On the stability of the linear functional equation, Proc. Nat. Acad. Sci. U.S.A. 27 (1941), 222-224.

[11] D. H. Hyers, G. Isac, Th. M. Rassias: Stability of Functional Equations in Several Variables, Birkhauser, Boston, Basel, Berlin, 1998.

[12] D. H. Hyers, Th. M. Rassias: Approximate homomorphisms, Aequationes Math. 44 (1992), no. $2-3,125-153$.

[13] S.-M. JunG: Hyers-Ulam-Rassias Stability of Functional Equations in Mathematical Analysis, Hadronic Press, Palm Harbor, Florida, 2001.

[14] I. Kaplansky: Modules over operator algebras, Amer J. Math. 75 (1953), 839-858.

[15] E. C. LancE: Hilbert $C^{*}-M o d u l e s$, LMS Lecture Note Series 210, Cambridge Univ. Press, 1995.

[16] H. Lin: Hilbert $C^{*}$-modules and their bounded module maps, Sci. China, Ser. A 34 (1991), no. $12,1409-1421$.

[17] H. Lin: Bounded module maps and pure completely positive mappings, J. Operator Theory 26 (1991), 121-138.

[18] V. M. Manuilov, E. V. Troitsky: Hilbert $C^{*}$-modules, Translations of Mathematical Monographs, 226. American Mathematical Society, Providence, RI, 2005.

[19] D. S. Mitrinović: Analytic inequalities, In cooperation with P. M. Vasić, Die Grundlehren der mathematischen Wissenschaften, Band 165, Springer-Verlag, New York-Berlin, 1970.

[20] Th. M. Rassias: On the stability of the linear mapping in Banach spaces, Proc. Amer. Math. Soc. 72 (1978), 297-300.

[21] Th.M. Rassias: On the stability of functional equations and a problem of Ulam, Acta Appl. Math. 62 (2000), no. 1, 23-130.

[22] Th. M. Rassias (ED.): Functional Equations, Inequalities and Applications, Kluwer Academic Publishers, Dordrecht, Boston, London, 2003.

[23] S. M. Ulam: Problems in Modern Mathematics, Chapter VI, Science Editions, Wiley, New York, 1964.

Michael Frank: Hochschule für Technik, Wirtschaft und Kultur (HTWK) Leipzig, Fachbereich Informatik, Mathematik und Naturwissenschaften (FbIMN), PF 301166, D-04251 Leipzig, Germany.

mfrank@imn.htwk-leipzig.de

Pasc Găvruţa: Department of Mathematics, University 'Politehnica' of Timişoara, Piata Victoriei, No. 2, 300006 Timişoara, Romania.

pgavruta@yahoo.com 
Mohammad Sal Moslehian: Department of Pure Mathematics, Ferdowsi University of Mashhad, P. O. Box 1159, Mashhad 91775, Iran;

Centre of Excellence in Analysis on Algebraic Structures (CEAAS), Ferdowsi University of Mashhad, Iran.

moslehian@ferdowsi.um.ac.ir and moslehian@ams.org 